\title{
Introduction to the Business Intelligence \& Big Data for Innovative and Sustainable Development of Organizations Minitrack
}

\author{
Celina M. Olszak \\ University of Economics in \\ Katowice \\ Poland \\ celina.olszak@ue.katowice.pl
}

\author{
Jozef Zurada \\ University of Louisville \\ USA \\ jozef.zurada@louisville.edu
}

\author{
Dilek Cetindamar \\ University of Technology Sydney \\ Australia \\ Dilek.ck@uts.edu.au
}

The development of the Internet, social media, distributed databases, and a variety of mobile devices has caused a huge increase in data. Much of this diverse data in unstructured and structured forms has a high business value and, if properly utilized, can become an important organizational asset. It contains various information about customers, competition, labor market, and development trends for industries, products and services, as well as the public and political mood. For innovative and sustainable development, it is essential for organizations to utilize this data to increase sales, identify future opportunities and new markets, outperform the competition, enhance products and services, recruit talent, improve operations, perform forecasting, protect the brand, and identify areas for improvement to name a few. However, many organizations make a limited use of this valuable data available to them either because they lack necessary tools or do not understand the value of this data. The main objective of this mini-track is to provide organizations a theoretical, conceptual, and applied grounded discussion of Business Intelligence and Big Data (BI \& BD) to aid in innovative and sustainable development, achieve competitive advantage as well as effective decision-making. This mini-track includes five papers, with the first paper nominated as the best mini-track paper.

In the first paper titled "Extending Loyalty Programs with BI Functionalities: A Case Study for Brick-andMortar Stores" by Kucklick et al. investigate how traditional brick-and-mortar stores can benefit from an expansion of service functionalities of a loyalty card provider. Their research was motivated by the fact that small sized brick-and-mortar stores have limited resources and cannot afford complex BI tools due to costs and maintenance constraints. Scaling these solutions for multiple shops via a common loyalty program makes the analytical service attractive and affordable. The loyalty program was transformed from simple card-based solution to a mobile app for customers and a web application for shop owners.

The new solution offers additional BI services for performing data analyses and strengthening the position of brick-and-mortar stores. Participating shops can collaborate to increase sales, align marketing campaigns, work together across industries, offer joint promotions and insights, plan workforce, and identify trends. The authors analyze shopping data from 12 years, 55 shops, and 19,000 customers.

The second paper titled "Crowdsourcing Contests: Understanding the Effect of Environment and Organization Specific Factors on Sustained Participation" by Khasraghi et al. identifies a research gap related to the environment-specific and organization-specific factors the influence individuals' sustained participation in the crowdsourcing contests. The authors conduct an empirical study using data from an online crowdsourcing contest platform, Kaggle, which delivers data science and machine learning solutions to its clients. Their research (1) shows the statistically significant effects of structural capital, familiarity with organization, and experience with the organization on individuals' sustained participation in crowdsourcing contests, (2) contributes to the literature by identifying the environment-specific and organization-specific factors that influence individuals' continued participation in crowdsourcing contests, and (3) offers guidance to organizations that host a crowdsourcing platform to design, implement, and operate successful crowdsourcing contest platforms.

In the third study entitled "Effects of Visualization Techniques on Understanding Inconsistencies in Automated Decision-Making" by Carl Corea et al., the authors present the results of an empirical experiment analyzing the cognitive effects of different 
visualization techniques for quantitative insights on understanding inconsistencies in automated decisionmaking data. They conduct and evaluate an empirical experiment using neuro- physiological measurement. The study shows that a ranking-based visualization is associated with a better understanding efficiency and lower mental effort in handling inconsistent cases. It also indicates that the visualization of big data insights is a key factor to create value from data for companies.

The fourth paper entitled "User Related Challenges of Self-Service Business Intelligence" by Christian Lennerholt et al. refers to a new trend in Business Intelligence named Self-service Business Intelligence (SSBI). The study explores user-related SSBI challenges by conducting and analyzing a total of 30 qualitative interviews with $5 \mathrm{BI}$ consultants and 10 customer representatives involved in 2 SSBI implementation project teams. Analysis of the interviews revealed ten challenges related to "Selfreliant users", seven challenges related to "creating SSBI reports" and five challenges related to "SSBI education" that differ considerably from SSBI challenges commonly discussed in literature.

Big Data (BD) has become a new source of competitive advantage because vast volumes of strategic, operational and tactical information consistently have been shared across the supply chain.
Therefore, supply chain managers started to heavily invest in sophisticated data analytics to leverage BD to extract and derive valuable insights with the potential to exceed traditional decision- making in order to achieve successful supply chain performance including sustainability. The impact of BD on sustainability is a neglected topic in literature. However, BD applications might induce many benefits for economic, environmental and social sustainability. For example, utilising BD technologies to analyse and understand firms' energy consumption behaviour, which leads to improve energy efficiency and promote energy conservation. This result better environmental and financial gains. In addition, it could further reduce various social problems such as improving workforce health and safety due to better energy and waste practices. Thus, the last paper titled "Assessing Big Data Analytics Capability and Sustainability in Supply Chains" by Cetindamar et al. aims to bridge two streams of studies in BD analytics and sustainable supply chain to show the link between them. Specifically, the paper explores what assessment metrics are available in literature that might be useful in future studies interested in examining this relationship in an empirical manner. In sum, the study seeks to address two research questions: (1) What capabilities are required to build BD analytics? and (2) What constitutes the dimensions of sustainable supply chains? 